Utah State University

DigitalCommons@USU

$1-1-2006$

\title{
Far-Infrared Spectroscopy of the Troposphere (FIRST): Flight Performance and Data Processing
}

\author{
S. Wellard \\ G. Bingham \\ H. Latvokoski \\ M. Mlynczak \\ D. Johnson \\ K. Jucks
}

Follow this and additional works at: https://digitalcommons.usu.edu/sdl_pubs

\section{Recommended Citation}

Wellard, S.; Bingham, G.; Latvokoski, H.; Mlynczak, M.; Johnson, D.; and Jucks, K., "Far-Infrared Spectroscopy of the Troposphere (FIRST): Flight Performance and Data Processing" (2006). Space Dynamics Lab Publications. Paper 139.

https://digitalcommons.usu.edu/sdl_pubs/139

This Article is brought to you for free and open access by the Space Dynamics Lab at DigitalCommons@USU. It has been accepted for inclusion in Space Dynamics Lab Publications by an authorized administrator of DigitalCommons@USU. For more information, please contact digitalcommons@usu.edu.

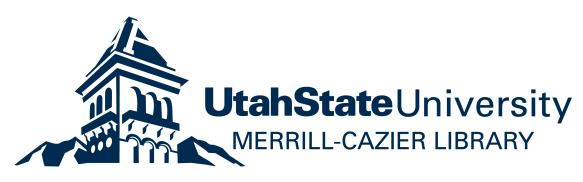




\title{
Far-infrared spectroscopy of the troposphere (FIRST): flight performance and data processing
}

\author{
S. Wellard ${ }^{\mathrm{a},}$, , G. Bingham ${ }^{\mathrm{a}}$, H. Latvakoski ${ }^{\mathrm{a}}$, M. Mlynczak ${ }^{\mathrm{b}}$, D. Johnson ${ }^{\mathrm{b}}$, K. Jucks ${ }^{\mathrm{c}}$ \\ ${ }^{a}$ Space Dynamics Laboratory, Utah State University, Logan, UT 84341-1947 \\ bASA, Langley Research Center, Hampton, VA 23681 \\ ${ }^{c}$ Harvard-Smithsonian Center for Astrophysics, Cambridge, MA
}

\begin{abstract}
The radiative balance of the troposphere, and hence global climate, is dominated by the infrared absorption and emission of water vapor, particularly at far-infrared (far-IR) wavelengths from 15-50 $\mu \mathrm{m}$. Current and planned satellites observe the infrared region to about $15.4 \mu \mathrm{m}$, ignoring spectral measurement of the far-IR region from 15 to $100 \mu \mathrm{m}$. The far-infrared spectroscopy of the troposphere (FIRST) project, flown in June 2005, provided a balloon-based demonstration of the two key technologies required for a space-based far-IR spectral sensor. We discuss the FIRST Fourier transform spectrometer system $\left(0.6 \mathrm{~cm}^{-1}\right.$ unapodized resolution), its radiometric calibration in the spectral range from 10 to $100 \mu \mathrm{m}$, and its performance and science data from the flight. Two primary and two secondary goals are given and data presented to show the goals were achieved by the FIRST flight.
\end{abstract}

Keywords: interferometer, far-infrared, calibration, water vapor, global energy balance

\section{INTRODUCTION}

The Far-Infrared Spectroscopy of the Troposphere (FIRST) instrument is an Imaging Fourier Transform Spectrometer (IFTS) developed at Utah State University's Space Dynamics Laboratory (SDL) as a NASA Langley Instrument Incubator Program (IIP). FIRST was designed to measure the infrared spectrum in the nadir view between 10 and 100 micrometers (1000 to 100 wavenumbers) at 0.6 wavenumber unapodized spectral resolution on a balloon platform from high altitudes $(\sim 35 \mathrm{~km})$. The instrument completed thermal vacuum testing and radiometric calibration during early 2005 and flew June 7, 2005 from the National Scientific Balloon Facility (NSBF) at Fort Sumner, New Mexico. The flight completed the technology demonstration and validation of the FIRST sensor. FIRST was designed to demonstrate two high priority climate measurements: calibrated radiances and the calculation of water vapor profiles of the upper troposphere and lower stratosphere using the far-IR.

\subsection{Significance}

The scientific case for directly measuring the far-infrared emission is reviewed by Mlynczak et al. (2002) ${ }^{1,2}$. We define the far-IR as wavelengths between 15 and $100 \mu \mathrm{m}$ because this portion of the Earth's emission spectrum is not directly observed from space despite its fundamental importance. Approximately one-half of the energy leaving the Earth is contained in this spectral region. One-half to three-quarters of the energy leaving the atmosphere is in the far-infrared. Figure 1 is an estimate ${ }^{3}$ of the ratio of the far-infrared flux at the top of the atmosphere to the total infrared flux at the top of the atmosphere using the NCAR Community Atmosphere Model (CAM). The CAM clearly illustrates that over half of the energy leaving the planet is in the far IR.

The far-IR is important for more reasons than total energy loss from the planet. Earth's climate is strongly influenced by radiative cooling associated with the emission of infrared radiation by water vapor at far-IR wavelengths extending out beyond $60 \mu \mathrm{m}$. The free troposphere cools radiatively almost exclusively in the far-IR. Water vapor is also the principal greenhouse gas, absorbing a significant fraction of the upwelling radiation from the Earth's surface and providing much of the downwelling longwave flux that warms the Earth's surface (i.e., the

Infrared Spaceborne Remote Sensing XIV, edited by Marija Strojnik, Proc. of SPIE

Vol. 6297, 62970Q, (2006) · 0277-786X/06/\$15 - doi: 10.1117/12.683976 
greenhouse effect). The distribution of water vapor and associated far-IR radiative forcings and feedbacks are well recognized as major uncertainties in predicting future climate.

Cirrus cloud systems modulate far-infrared radiation, especially in the tropical upper atmosphere, implying that cirrus clouds play an important role in climate ${ }^{4}$. The effects of cirrus clouds in attenuating the far-IR to $25 \mu \mathrm{m}$ have been shown by the Russian Meteor spacecraft. ${ }^{5}$. Spectral measurements of the far-IR may also offer the potential for increased accuracy in water vapor profiles retrieved from emission measurements ${ }^{6}$. Far-infrared measurements also offer the potential for determining the optical properties of cirrus particles ${ }^{7}$. Measurements of the far-IR will provide basic information about the Earth's atmosphere and climate system and contribute significantly to understanding how it responds to various natural and human inputs.

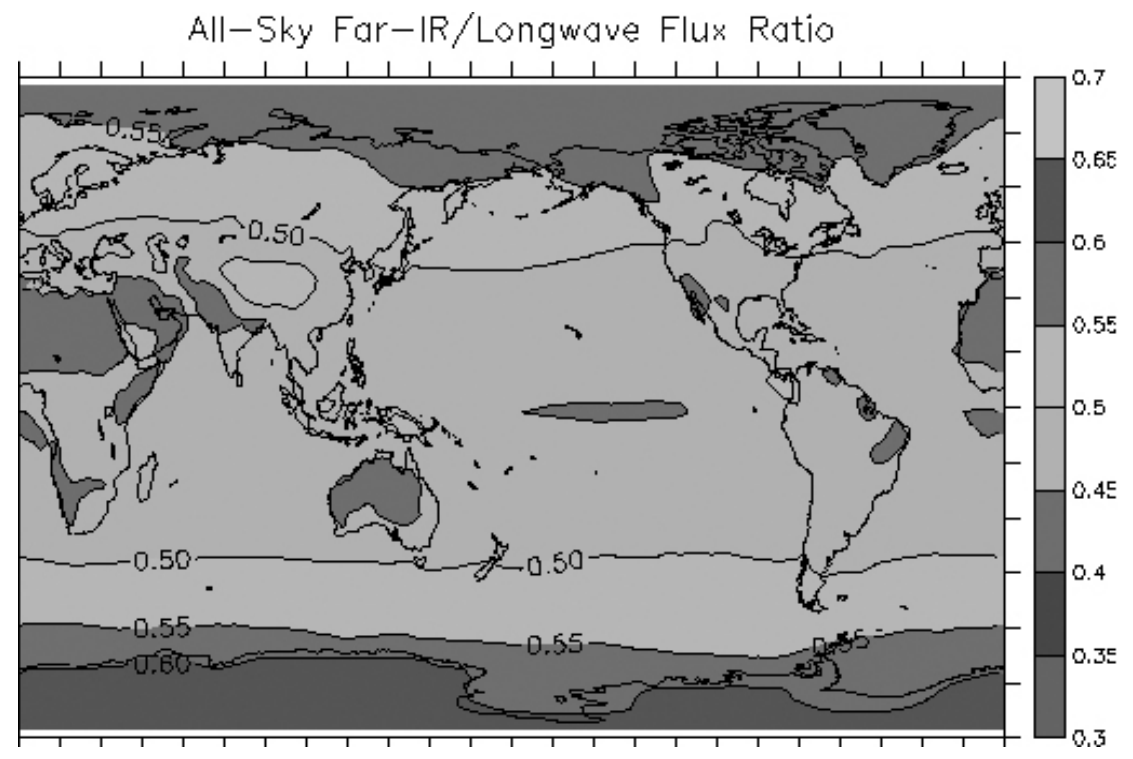

Figure 1. Ratio of the top-of-atmosphere far-infrared flux to total infrared flux as computed by the NCAR Community Atmosphere Model.

\section{THE FIRST INSTRUMENT: DESCRIPTION AND TECHNOLOGY}

The mechanical model and optical path of the FIRST sensor are shown in Figure 2. The sensor has three major subassemblies: a scene select mirror assembly, the main Dewar housing a high-throughput Michelson interferometer and aft optics, and a second Dewar containing the sensor's detectors. The three sections are separated by dual polypropylene windows, one between the scene mirror assembly and the main Dewar with the other between the main and detector Dewars. The windows allow the sections to operate independently at different temperatures and pressures. The scene mirror section is open to ambient; the main Dewar is cooled with LN2 and controlled to $180 \mathrm{~K}$ to simulate passive on-orbit cooling and reduce background. The detector Dewar was cooled to $4.2 \mathrm{~K}$ with liquid helium. 



Figure 2. (A) The FIRST sensor model showing the major components and the selectable measurement and calibration views. (B) The optical path through the sensor, with the scene selection mirror looking into the warm, deep cavity, far IR blackbody.

The scene mirror, under computer control, alternated between three available views. The primary view is nadir looking down at the Earth and its atmosphere. Periodically the mirror is rotated to view the on-board calibration blackbody and then the high elevation space view. The data collected from these two views was used post flight to reduce main channel interferometric data.

The Mickelson interferometer and its wide-band beam splitter were the emphasis of the FIRST sensor design. The interferometer is a plane-mirror Michelson design featuring a throughput of $0.47 \mathrm{~cm}^{2} \mathrm{sr}$ with a beam diameter of 7 $\mathrm{cm}$. Previous SDL designs had beam diameters of $5 \mathrm{~cm}$. The moving mirror of the interferometer scanned over a double-sided optical path difference of $\pm 0.8 \mathrm{~cm}$ to give a nominal resolution of $0.625 \mathrm{~cm}^{-1}$. The scan period during flight was 8.6 seconds although it could have been set to any period between 8.6 and 1.4 seconds depending on desired detector sample intervals.

The broadband beam splitter integrated into the FIRST interferometer design was based on research described by Dobrowolski and $\mathrm{Traub}^{8}$. The beam splitter was fabricated using an electron beam evaporation technique to deposit approximately 1.05 microns of germanium onto a 3.5-micron polypropylene film. Polypropylene was selected as the substrate material because it has few absorption features in the passband. Figure 3 shows the flight beam splitter and the reflection/transmission (RT) functions. 


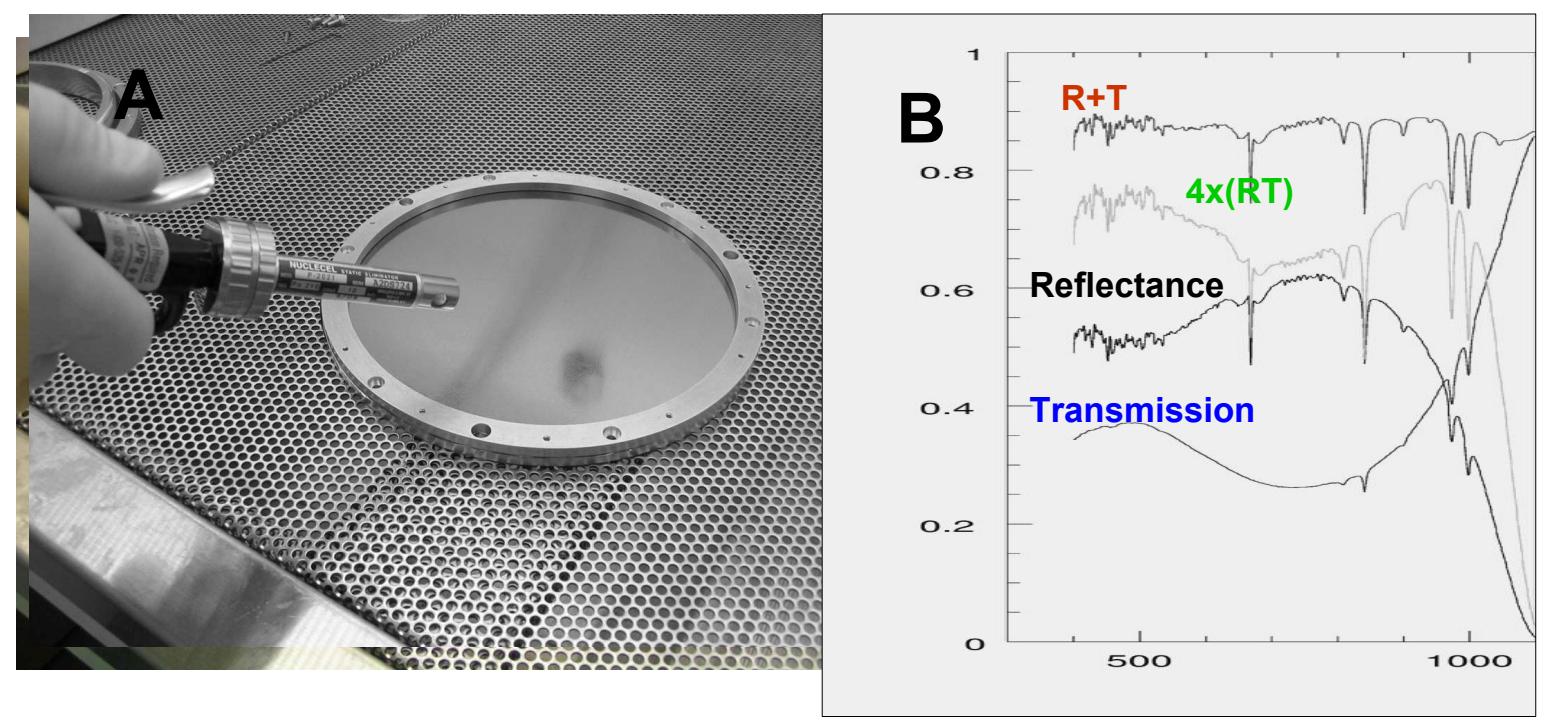

Figure 3. (A) The FIRST beamsplitter being prepared for mounting into the interferometer cube. Structures in the beamsplitter are reflections of the clean bench ceiling. (B) RT curves for a typical beamsplitter showing absorption as a function of wavenumber.

The clear diameter of the finished beam splitter is $17.8 \mathrm{~cm}$ reduced to $12.8 \mathrm{~cm}$ when mounted in the interferometer. The performance of the flight beam splitter was tested with the Smithsonian Astrophysical Observatory FIRS-4 spectrometer ${ }^{9}$ to insure proper wavelength response over the 100 to $1000 \mathrm{~cm}^{-1}$ operational band of the FIRST sensor.

Because of concerns about placing a clear area in the main beamsplitter to pass the beam of the helium-neon laser, the metrology interferometer was designed as an independent interferometer, only sharing the moving mirror of the signal interferometer. The metrology interferometer, using a 0.6328-micron Helium-Neon laser as its signal input, had its own separate "fixed-mirror," beam splitter, and detector.

The collimated output of the signal interferometer was focused by aft optics onto the array of Winston cone flux concentrators in the sensor Dewar. The cones direct infrared signal energy to the corresponding discrete microbolometer set into individual integration cavities. The focal plane concept for a FIRST satellite sensor proposes 100 Winston cone/micro-bolometers arranged in a 10 X 10 pattern. This gives a $100-\mathrm{km}$ square cross-track footprint on the surface of the Earth from a typical satellite altitude. For the demonstration, only 10 Winston cone/microbolometers were installed on the $3.75-\mathrm{cm}$ square focal plane (two detectors at each corner and two in the center). The flight interferometer with its high throughput optics and beam splitter is shown in Figure 4. 


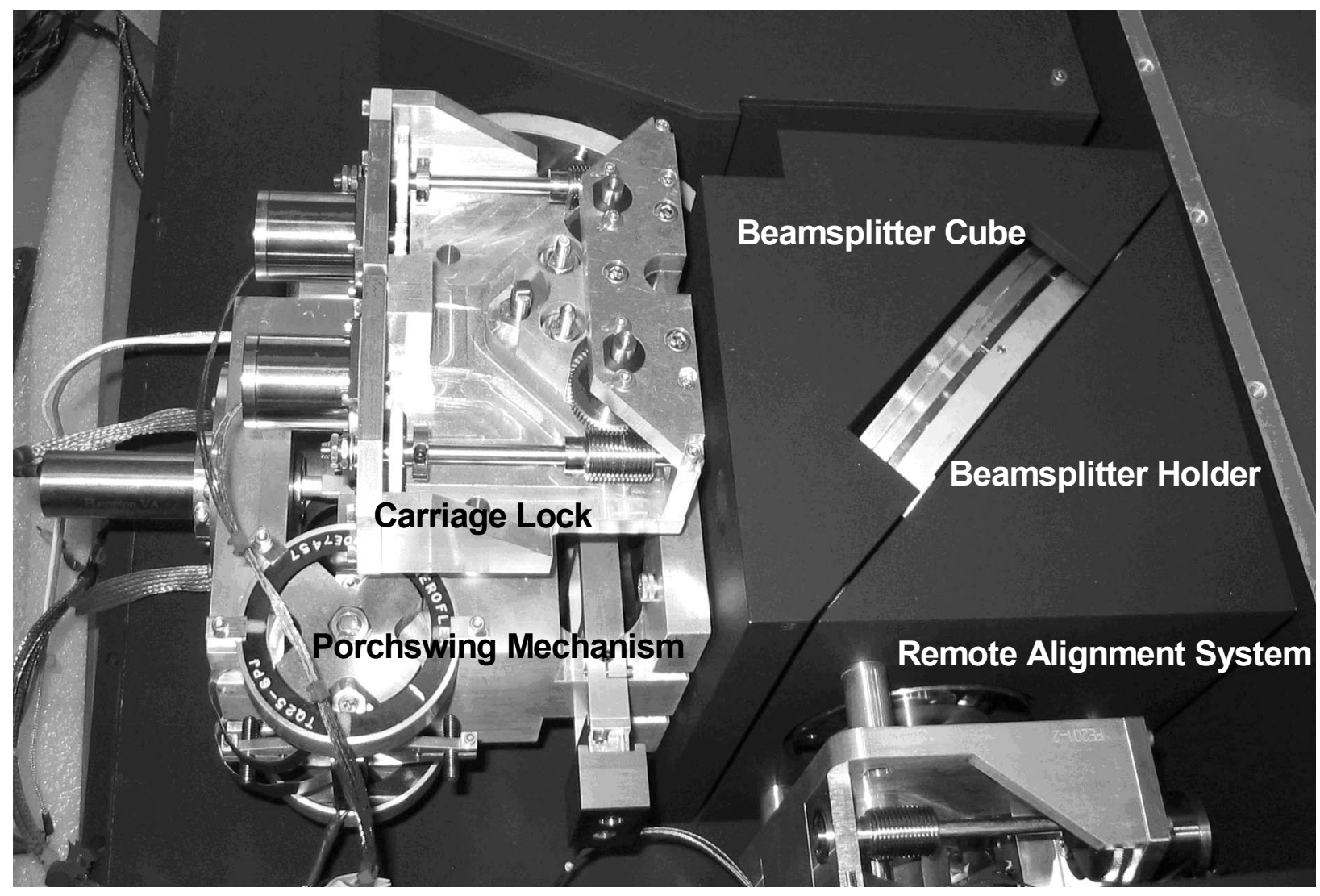

Figure 4: SDL High Throughput Interferometer

\section{THE FIRST INSTRUMENT: TECHNOLOGY PERFORMANCE GOALS}

The FIRST FTS was tasked to meet two primary and two secondary goals in the course of the FIRST program. The first primary goal was a demonstration of a high-throughput interferometer capable of fully illuminating the entire focal plane. The next goal was the successful design, fabrication, and integration of a pellicle beam splitter capable of satisfactory performance over the full 10 to $100 \mu \mathrm{m}$ range of the FTS. After demonstrated performance, the technology readiness levels (TRLs) for these two technologies would increase and the sensor would be ready to go to space as a satellite-based instrument.

Secondary design goals included a demonstration of sensor performance with a passive thermal system operating at $180 \mathrm{~K}$ and, finally, the achievement of sensor noise equivalent temperature differences (NETDs) of, at most, $0.5 \mathrm{~K}$ over a range of 100 to $170 \mathrm{~cm}^{-1}$ and $0.2 \mathrm{~K}$ from 170 to $1000 \mathrm{~cm}^{-1}$.

As described in the following sections, the performance of the FIRST sensor met the four goals set at the beginning of the program.

\section{FIRST CALIBRATION}

SDL dedicated significant resources to the calibration and characterization of the FIRST sensor before flight. Calibration characteristics were designed into the FIRST system from the beginning, with the detailed calibration efforts developed for the GIFTS and CrIS sensors serving as a baseline for the FIRST calibration plan. Calibrating FIRST required addressing basic issues of absolute radiometric response, frequency scale, instrument line shape, phase alignment and self-apodization. Techniques for quantifying and modeling these effects were applied to FIRST $^{10,11,12,13,14,15}$ during its calibration. For Earth scene calibration, FIRST utilized the same calibration scheme adopted by the NPOESS Cross Track Infrared Sounder (CrIS) sensor with calibration information from space view (cold BB) and then the warm flight blackbody. Spectra from each source were averaged and applied to the Earth 
spectra during the absolute calibration process. Using this technique, the University of Wisconsin has recently shown calibration agreement between the aircraft mounted S-HIS and the AQUA AIRS sounder of about $0.1 \mathrm{~K}^{16,17}$.

\subsection{Calibration Approach}

The FIRST calibration measured system dark currents, trends, SNR, transient behavior, absolute responsivity, linearity correction, and point response measurements for wavelength scale verification and instrument line shape. The FTS system used in FIRST has a well-understood instrument line shape (ILS), depending mainly on the wavelength of the laser used for sampling and the geometry of the focal plane relative to the interferometer axis. System linearity correction is critical for sounder spectral measurements. This was measured using a small signal response vs. signal level data set collected with SDL's Multifunction Infrared Calibrator version 2 (MIC 2) ${ }^{18,19}$.

Figure 5(A) shows spectra from detector 1 in the forward direction of the warm blackbody taken about an hour and a half apart. These spectra are a combination of $\sim 20$ individual scans. The plots show magnitude and phase. The wavenumber resolution is 0.643 wavenumbers. The phases are different because of slow shifts in the relative spacing of the laser and IR interferometers. As this type of change in phase is linear with wavenumber, it is easy to align the phases. The magnitudes vary by up to 0.004 of the units shown here over a day, and more from day to day, while the shape of the phase curves do not change significantly over time. Backgrounds (SVS scans) show a similar variation in magnitude and more variation in the shape of the phase curves. The spectra extend to 7901 wavenumbers, but there are no useful data above the range shown here. The wiggles in the spectra with a period of $\sim 100$ wavenumbers are due to the polypropylene windows.

Figure 6(B) shows spectra of the warm blackbody and the space view simulator. As in (A), these are combinations of 20 scans from detector 1 in the forward direction. The phases do not match because out of phase light is present (which may be due to beam splitter emission). Because of the previously mentioned variation on interferometer optical paths, these phases must be aligned to the correct relative position before spectra can be background subtracted. Since these curves will never overlap, the proper match is not obvious. For the WBB spectra, the phase alignment is found (to within $\sim 0.5^{\circ}$ ) by comparing spectra of the WBB and Space View Source (SVS) taken immediately after each other. (The data shown here have been aligned.) The phases for scans of the variable temperature Long Wave IR Calibration Source (LWIRCS) at various temperatures are aligned by making these cross where the phases of the WBB and SVS scans cross.
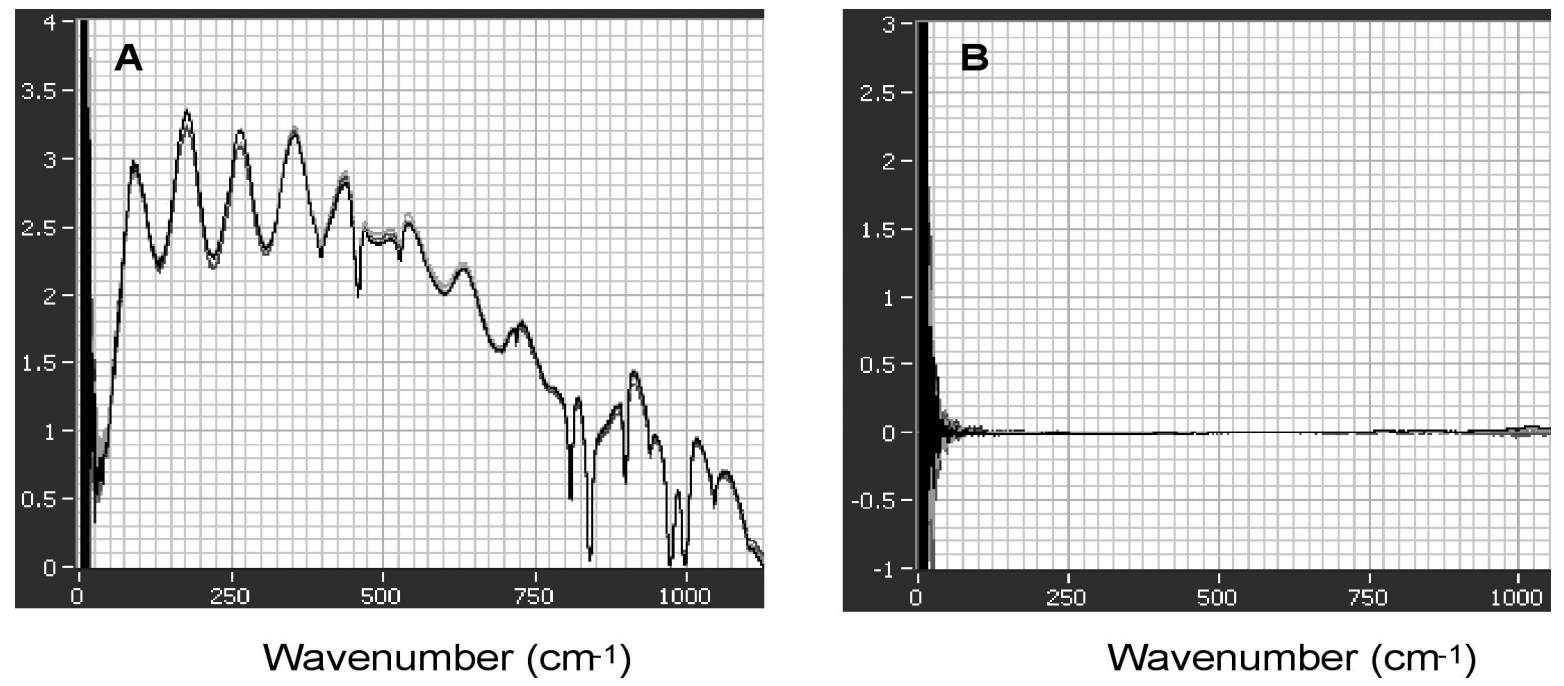

Figure 5. (A) System stability, shown by the real responsivity of detector 1 looking at a warm blackbody calculated for 9 spectra collected over a 2-day period. (B) The imaginary responsivity of the same spectra. 

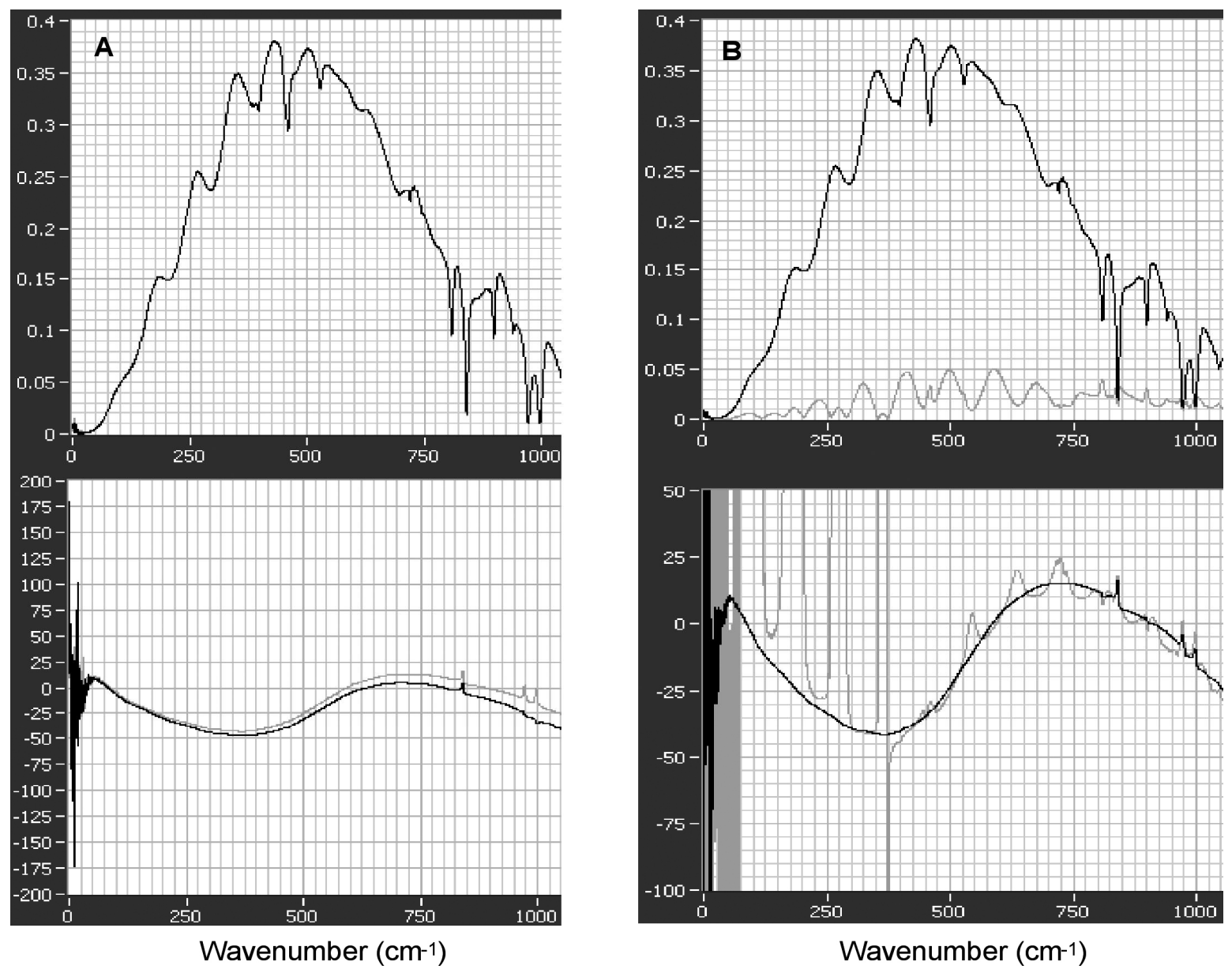

Figure 6. (A) Relative response and phase of 20 scans of two spectra of the warm BB collected 1.5 hours apart. (B) The relative response and phase from the warm and space simulation BBs showing the phase differences.

Figure 7(A) shows the radiance from LWIRCS at a variety of temperatures as measured by FIRST. These spectra are background subtracted, phase corrected and divided by the responsivity curve. The data used here are from detector 1 collected in the forward direction. The structures in the curves (noise spikes) result from errors in the responsivity curves due to small values where the beam splitter transmission is low. Figure 7(B) shows the resulting brightness temperatures of LWIRCS as measured by FIRST. They are calculated by using the inverse Planck function on the radiance curves.

Figure 7(B) shows that FIRST is meeting its significant performance specifications. The temperature error is dominated by broad systematic variations. The high frequency variations (the barely visible fuzz) are at a level of $0.1 \mathrm{~K}$ peak to peak between 250 and 950 wavenumbers. This corresponds to an rms error of approximately $0.1 \mathrm{~K}$ in an individual scan, thus meeting the sensitivity goal. 

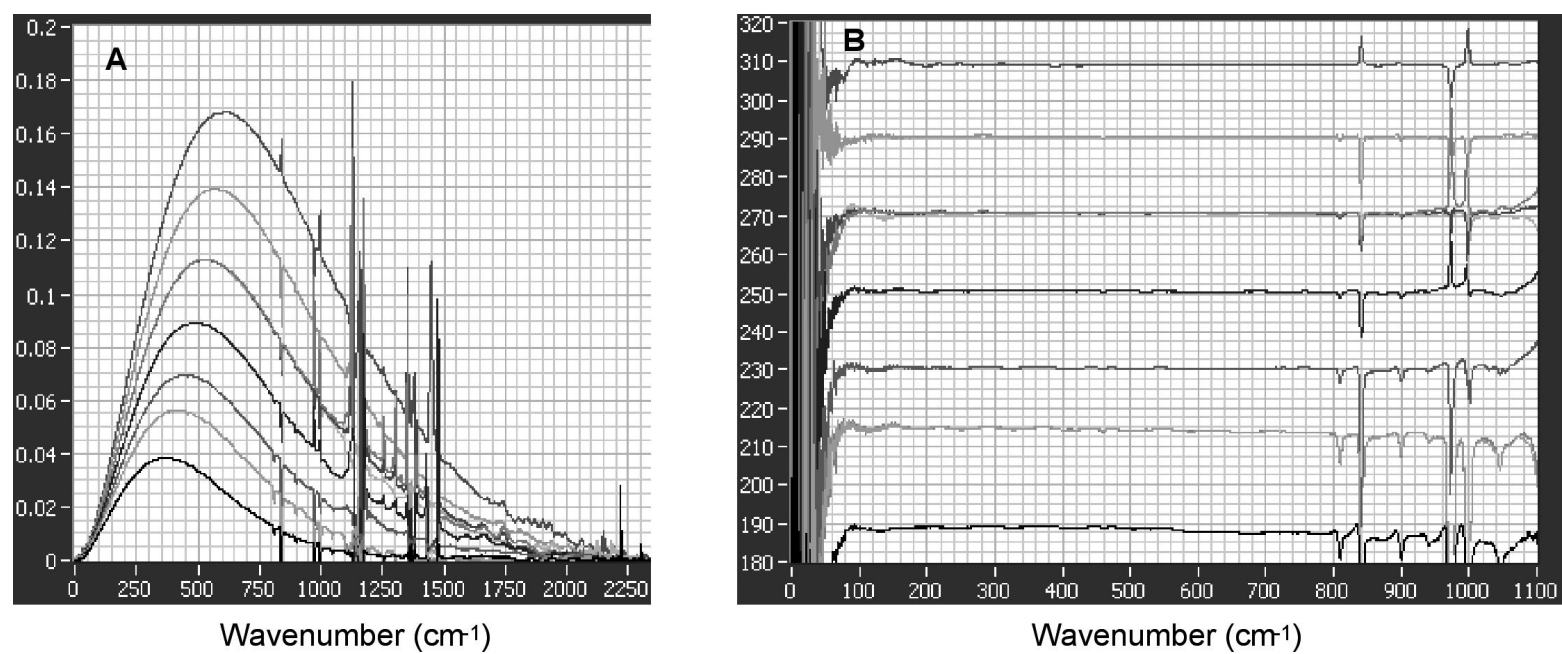

Figure 7. (A) Calibrated Radiance response with the LWIRCS at various temperatures. Structures are the results from error due to small values in the responsivity curves where the beam splitter transmission is low. (B) The spectral temperature response curves resulting from dividing the curves in (A) by their inverse Plank functions.

\section{FLIGHT SPECTRA}

The FIRST sensor was launched from Fort Sumner, New Mexico at about 8 A.M. local time on June 7, 2005 and remained at altitude until "cut-down" at about 6 P.M. The payload floated to an altitude of approximately $27 \mathrm{~km}$ in about an hour and operated there for the next 5.5 hours collecting approximately 15000 interferograms. The operational script called for FIRST to look down at the Earth, upper troposphere and the lower stratosphere for 15 minutes. The scan mirror then moved to the cold space view for 5 minutes and then to the reference blackbody for 5 minutes. Because wind velocities aloft were low during the flight, the sensor remained within $80 \mathrm{~km}$ of the launch point.

Figure 8 shows two 50 to $2000 \mathrm{~cm}^{-1}$ spectrum recorded on a central detector (A) and a corner detector (B) that demonstrate the high throughput capabilities of the FIRST FTS and the wide-band performance of the beam splitter. The data above $1000 \mathrm{~cm}^{-1}$ are plotted with gaps that are inserted when significant absorption reduced the signal below a point that could be accurately calibrated. Metric Planck functions are super-imposed on (A) and (B) to allow estimates of brightness temperatures.
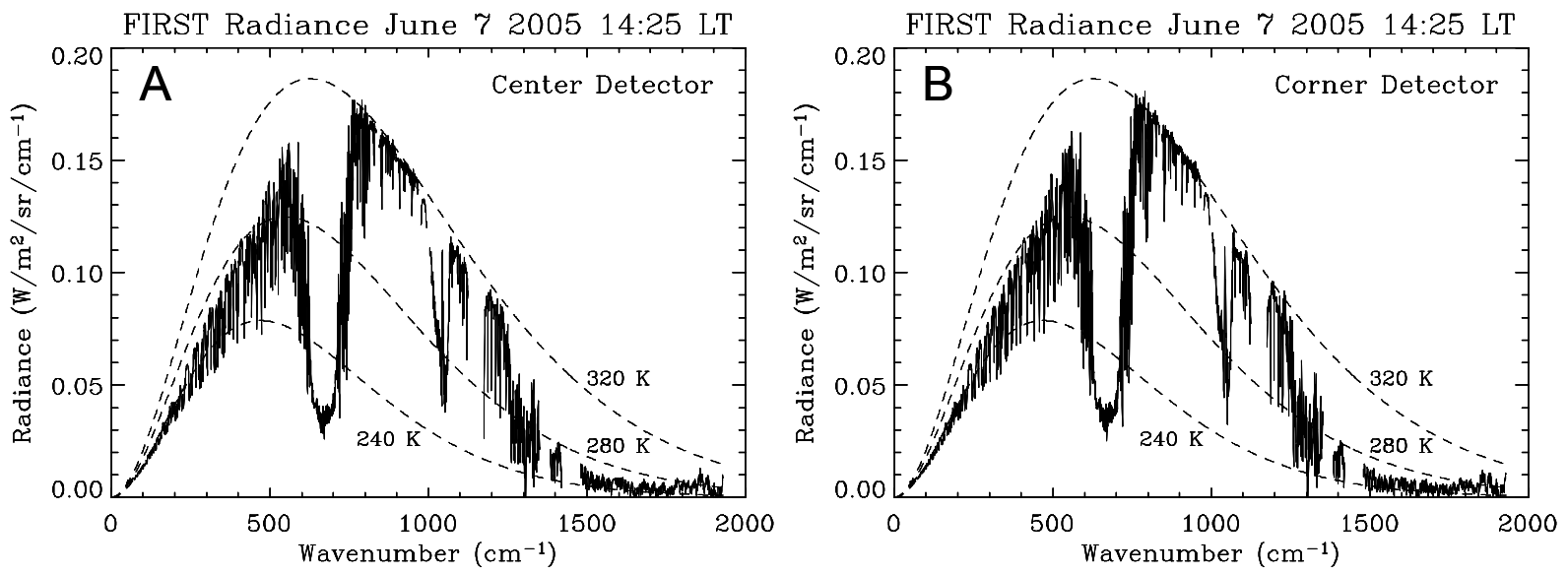

Figure 8. Two flight spectra collected at the time of the Aqua overpass. (A) is a center detector and (B) a corner detector. The transformed spectra are compared with BB spectra with the labeled temperatures. 
An initial assessment of the calibration accuracy of the FIRST radiances is made by looking at the atmospheric window region ( 800 to 1000 wavenumbers) and comparing these with the measurements recorded by instruments on the Aqua satellite. The FIRST radiance at 900 wavenumbers $\left(\mathrm{cm}^{-1}\right)$ of $0.15 \mathrm{~W} \mathrm{~m} \mathrm{sr}^{-1}\left(\mathrm{~cm}^{-1}\right)^{-1}$ corresponds to a blackbody surface skin temperature of 318 Kelvin. At first this seems quite high, but the FIRST flight occurred in cloudless skies on a warm day with air temperatures above $32^{\circ} \mathrm{C}$ or 305 Kelvin. The radiance recorded by the CERES instrument in its window channel (covering 877 to 1227 wavenumbers, spectrally integrated) was $41.75 \mathrm{~W}$ $\mathrm{m}^{-2}$ sr. Line by line radiative transfer calculations (e.g., Kratz et al., $2005^{20}$ ) simulating the CERES measurement and incorporating surface temperatures of $318 \mathrm{~K}$ and $297 \mathrm{~K}$ with an atmospheric temperature profile from a radiosonde launched at Albuquerque, New Mexico, yielded radiances of $41.83 \mathrm{~W} \mathrm{~m}^{-2} \mathrm{sr}^{-1}$ and $30.76 \mathrm{~W} \mathrm{~m}^{-2} \mathrm{sr}^{-1}$, respectively, confirming the surface temperature of $318 \mathrm{~K}$ derived from FIRST and therefore the ground calibration of FIRST.

A direct comparison of surface skin temperature is available using data from the AIRS instrument. The AIRS database records 4 fields-of-view that are centered $52 \mathrm{~km}, 46 \mathrm{~km}, 37 \mathrm{~km}$ and $77 \mathrm{~km}$ from the location of FIRST. The skin temperatures in the 4 fields are $318.5 \mathrm{~K}, 314.8 \mathrm{~K}, 319.0 \mathrm{~K}$, and $311.4 \mathrm{~K}$, respectively. The AIRS observation closest to the FIRST location is within a degree of the FIRST observation. A key point to remember is that the AIRS field of view is about 4900 times larger than the FIRST field of view (14 km diameter vs. $0.2 \mathrm{~km}$ diameter.) With these considerations, these results show that FIRST is accurately calibrated, perhaps to better than $1 \mathrm{~K}$.

Since the focus of FIRST is on the far-infrared, we show in Figure 9 a FIRST spectrum between the limits of 40 and 600 wavenumbers. Also shown is a spectrum computed with a line-by-line radiative transfer code (at 0.625 wavenumber resolution) using as inputs the coincident profiles of temperature and moisture from AIRS. The calculated spectrum is offset by an amount equal to -0.05 radiance units, allowing visual inspection and comparison of the spectral structure of the FIRST measurements against the calculation. The FIRST data are a single measured spectrum, i.e., no averaging of multiple spectra has been performed. As is clearly evident, FIRST picks up the fine features in the spectra predicted by radiative transfer theory across the entire far-infrared. The data show remarkable fidelity with theory in the overall magnitude and structure of the far-infrared.

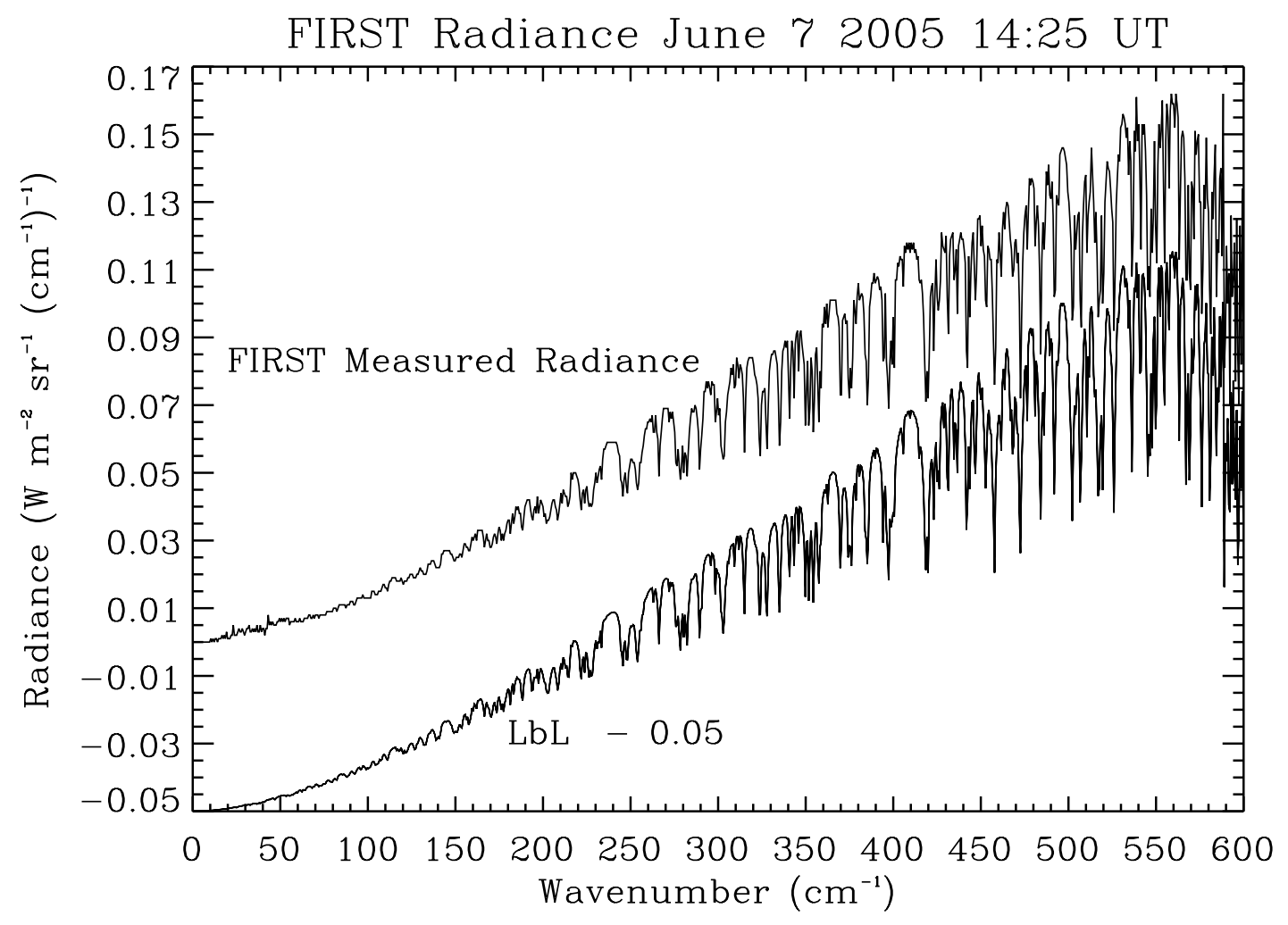

Figure 9. Far-infrared spectrum ( 80 to 600 wavenumbers) measured by FIRST (top curve) and line-by-line radiative transfer calculations based on AIRS soundings (bottom curve, offset by -0.05 radiance units), demonstrating spectral fidelity of the FIRST measurement relative to theory. 


\section{CONCLUSION}

FIRST, a Fourier Transform Spectrometer developed by NASA and SDL under the NASA Instrument Incubator Program, is a demonstration instrument for future, far-IR space based sensors. FIRST was designed to measure the infrared spectrum in the nadir view between 10 and 100 micrometers (1000 to 100 wavenumbers) at 0.6 wavenumber unapodized spectral resolution from a high altitude $(35 \mathrm{~km})$ balloon platform. The instrument was subjected to an intensive thermal vacuum and calibration exercise and flew on a high altitude balloon from Fort Sumner, New Mexico. Reduced flight data show FIRST successfully completed its technology demonstration and validation under the IIP program to meet its two primary program goals. Analysis of calibration data indicates that FIRST achieved its $0.2 \mathrm{~K}$ sensitivity goal and, in meeting the other three goals, meets the passive cooling goal as well.

The FIRST team anticipates future deployments of the payload in both flight and ground based campaigns in order to study many aspects of the Earth's climate including water vapor feedbacks, cirrus radiative properties (in the midand far-infrared simultaneously), and the natural greenhouse effect of the planet.

\section{REFERENCES}

1. Mlynczak, M. G., et al., "The Far-Infrared: A frontier in the remote sensing of Earth's climate and energy balance," SPIE, Vol. 4485, 220-224, 2002.

2. Martin G. Mlynczak, David G. Johnson, Harri M. Latvakoski, Kenneth W. Jucks, Mike Watson, David P. Kratz, Gail Bingham, Wesley A. Traub, Stanley J. Wellard, Charles R. Hyde, and Xu Liu, "First light from the FAR-Infrared Spectroscopy of the Troposphere (FIRST) instrument," Geophys. Res. Lett, 33, L07704,doi:10.1029/2005GL025114, 2006.

3. Collins, W. D., and M. G. Mlynczak, "Prospects for measurement of far-infrared tropospheric spectra: Implications for climate modeling," Fall Meeting, American Geophysical Union, San Francisco, December, 2001.

4. Liou, K.-N., "Influence of cirrus clouds on weather and climate processes: a global perspective," Mon. Wea. Rev., 114, pp. 1167-1199, 1986.

5. Spankuch, D. and W. Dohler, "Radiative properties of cirrus clouds in the middle IR derived from Fourier spectrometer measurements from space," Z. Meteorol., 35, pp. 314-324, 1985.

6. Mertens, C. J., "Feasibility of retrieving upper tropospheric water vapor from observations of far-infrared radiation," Optical Spectroscopic Techniques, Remote Sensing, and Instrumentation for Atmospheric and Space Research IV, Allen M. Larar and Martin G. Mlynczak, Editors, Proceedings of SPIE Vol. 4485, 191-201, 2002.

7. Yang, P. M. Mlynczak, H.L. Wei, D. P. Kratz, B. A. Baum, Y. X. Hu, A. Heidinger, and M. Mishchenko, "Spectral signature of cirrus clouds in the far-infrared region: Single-scattering calculations and radiative sensitivity study,” J. Geophys. Res., Vol. 108, No. D18, 4569, doi: 10.1029/2002JD003291, 2003.

8. Dobrowolski, J.A. and W.A. Traub, New Designs for Far-Infrared Beamsplitters, Applied Optics, 35, 2934, 1996.

9. Johnson, D. G., K. W. Jucks, W. A. Traub and K. V. Chance, "Smithsonian stratospheric far-infrared spectrometer and data reduction system," J. Geophys. Res., 100, 3091-3106, 1995.

10. Bingham, G. E., D. K. Zhou, B. Y. Bartschi, G. P. Anderson, D. R. Smith, J. H. Chetwynd and R. M. Nadile, "CIRRIS-1A Calibration, Earthlimb spectral measurements, and Atmospheric O3, HNO3, CFC-12, CFC-11 Profile Retrieval,” J. Geophysical Res. Vol. 102, No. D3, p 3547-3558, 1997.

11. Bingham, G. E., Harri M. Latvakoski, Stanley J. Wellard, Martin G. Mlynczak, David G. Johnson, Wesley A. Traub, and Kenneth W. Jucks, "Far-infrared spectroscopy of the troposphere (FIRST): sensor calibration performance," SPIE Fourth International Asia-Pacific Environmental Remote Sensing Symposium. 
Multispectral and Hyperspectral Remote Sensing Instruments and Applications II. Paper 5655-25. Honolulu, HI, USA, November 8-11, 2004.

12. Zhou, D. K., M. G. Mlynczak, G. Bingham, J. Wise, and R. Nadile, Geophys. Res. Lett., 25, 643-646, 1998.

13. Best, F. A., H. E. Revercomb, R. O. Knuteson, D. C. Tobin, S. D. Ellington, M. W. Werner, D. P. Adler, R. K. Garcia, N. N. Ciganovich, W. L. Smith, Sr., G. E. Bingham, J. D. Elwell, D. K. Scott, "The Geosynchronous Imaging Fourier Transform Spectrometer (GIFTS) onboard blackbody calibration system," SPIE Remote Sensing of the Atmosphere, Ocean, Environment and Space, 5655-10, Honolulu, HI , November 8-12, 2004.

14. Elwel1, John D., Deron K. Scott, Henry E. Revercomb, Fred A. Best, and Robert O. Knuteson, "Ground and On-Orbit Characterization and Calibration of the Geosynchronous Imaging Fourier Transform Spectrometer (GIFTS)," USU/SDL CALCON, Logan, Utah, September 16-18, 2003.

15. Knuteson, R. O., F. A. Best, G. E. Bingham, J. D. Elwell, H. E. Revercomb, D. C. Tobin, D. K. Scott, W. L. Smith, "On-orbit calibration of the Geosynchronous Imaging Fourier Transform Spectrometer (GIFTS)," SPIE Remote Sensing of the Atmosphere, Ocean, Environment and Space, 5655-09, Honolulu, HI, November 8-12, 2004.

16. Revercomb, H. E., D. Tobin, R. O. Knuteson, F. Best, W. Smith, D. D. LaPorte, S. D. Ellington, M. W. Werner, R. K. Garcia, N. N. Ciganovich, J. K. Taylor, E. R. Olson, B. Howell, "Highly accurate FTIR observations from the scanning HIS aircraft instrument," SPIE Remote Sensing of the Atmosphere, Ocean, Environment and Space, 5655-07, Honolulu, HI, November 8-12, 2004.

17. Revercomb, Hank, David C. Tobin, Robert O. Knuteson, Fred A. Best, William L. Smith, Paul van Delst, Daniel D. LaPorte, Scott D. Ellington, Mark W.Werner, Ralph G. Dedecker, Ray K. Garcia, Nick N. Ciganovich, H.Benjamin Howell, and Steven Dutcher, "Scanning HIS Aircraft Instrument Calibration \& AIRS Validation,” USU/SDL CALCON, Logan, Utah, September 16-18, 2003.

18. Tansock, J., A. Thurgood, R. Esplin, "Focus Optimization of a Cryogenic Collimator using Interferometric measurements and Optical Modeling," Proceedings of SPIE - The International Society of Optical Engineering, Vol. 2268, pp. 196-206, July, 1994.

19. Blakeley, J., "Improved IR Sensor Characterizations Using SDL/USU Multifunction Infrared Calibrators," Sixth SDL/USU Symposium on Infrared Radiometric Sensor Calibration, May, 1996.

20. Kratz, D. P., M. G. Mylnczak, H. Brindley, L. L. Gordley, F. Martin-Torres, F. M. Miskolczi and D. D. Turner, "An inter-comparison of far-infrared line-by-line radiative transfer models," J. Quant. Spectrosc. Radat. Transfer, 90, 323-341, 2005. 